\title{
Incorporación del pensamiento crítico en la enseñanza del lenguaje
}

\author{
Incorporation of Critical Thinking in Language Teaching
}

Incorporação do pensamento crítico no ensino da linguagem

\author{
Fernando Romero Ámbito \\ Magíster en Gestión de la Tecnología Educativa \\ Corporación Universitaria Minuto de Dios \\ ambito79@hotmail.com
}

\section{Resumen}

La enseñanza del lenguaje concede a los individuos que se forman en el sistema educativo la oportunidad de comunicarse a partir de un complejo entramado de mecanismos, a través de los cuales pueden transmitir conceptos de múltiples diversidades y composiciones. Sin embargo, esta enseñanza ha dejado, históricamente, de fortalecer el pensamiento crítico de quienes acceden a su formación. Este documento es fruto de un ejercicio reflexivo que intenta brindar estrategias para incorporar el pensamiento crítico, inspirado principalmente en el pensamiento del brasileño Paulo Freire, a la enseñanza de lenguaje en los niveles más generales, sin transformar completamente sus mecanismos, a la vez que brinda posibilidades de transformación de la manera en que los estudiantes perciben el mundo e interactúan con él a través de la comunicación.

Palabras clave: Lenguaje, pensamiento crítico, educación, estrategia

\begin{abstract}
Abstrac
The teaching of language gives individuals who are formed in the educational system the opportunity to communicate through a complex
\end{abstract}


network of mechanisms through which they can transmit concepts of multiple diversities and compositions. However, this teaching has historically ceased to strengthen the critical thinking of those who access its formation. This document is the result of a reflective exercise that seeks to provide strategies to incorporate critical thinking, inspired by the legacy of Paulo Freire, into language teaching at the most general levels, without completely transforming its mechanisms, at the same time it offers possibilities for transforming the way students perceive the world and interact with it through communication.

Keywords: Language, critical thinking, education, strategy.

\section{Resumo}

O ensino da linguagem proporciona aos indivíduos que se formam no sistema educacional à oportunidade de se comunicarem por meio de uma complexa rede de mecanismos, por meio dos quais podem transmitir conceitos de múltiplas diversidades e composições. Porém, historicamente, esse ensino tem falhado em fortalecer o pensamento crítico de quem acessa sua formação. Este documento é o resultado de um exercício reflexivo que tenta fornecer estratégias para incorporar o pensamento crítico, inspirado principalmente no pensamento do brasileiro Paulo Freire, ao ensino da linguagem nos níveis mais gerais, sem transformar completamente seus mecanismos, ao mesmo tempo, proporciona possibilidades de transformação na forma como os alunos percebem o mundo e interagem com ele por meio da comunicação.

Palavras-chave: Linguagem, pensamento crítico, educação, estratégia.

\section{El lenguaje como conducto de ideas}

Desde que el ser humano ha sido capaz de registrar los acontecimientos que definen y transforman su entorno, el lenguaje se ha convertido en la herramienta indispensable a través de la cual se comparte, se define y se transforma el devenir de la vida misma. De acuerdo con las definiciones ofrecidas por la RAE (Real Academia Española), el concepto en sí mismo es polisémico, ya que posee, por lo menos, siete definiciones, dependiendo del contexto en el que se trabaje. No obstante, llama la atención el hecho de que la quinta definición indica que el lenguaje es un "Conjunto de señales que dan a entender algo" (RAE, 2021).

De esta definición se puede deducir que la esencia del lenguaje no se reduce a un acto comunicativo tal como lo define el filósofo John Searle (1995), quien 
afirma que el lenguaje es un elemento constitutivo de los hechos sociales ${ }^{1}$, sino como una manera de estructurar elementos abstractos que se pueden compartir con el exterior, lo que comúnmente se conoce como una idea.

Esta capacidad del lenguaje de transmitir información, tanto si esta tiene sustento en elementos materiales como si es una representación abstracta, le permite ser concebido como una herramienta ideal para la creación, desarrollo y transmisión de complejas estructuras argumentativas, las cuales pueden orientar el curso de otros aspectos importantes en la interacción humana, tales como las relaciones de poder y los actos políticos.

Dicho de manera sencilla, el lenguaje, conceptualizado como un conducto de ideas, es la manera más apropiada de poner en elementos comprensibles un conjunto de elementos que no tienen otro modo de manifestarse en el mundo real, ni de tener aplicaciones prácticas. A su vez, esta característica justifica su importancia para el ser humano, y crea la necesidad de su enseñanza como parte vital de la formación de nuevos integrantes de la sociedad.

1 El concepto refiere a un postulado durkheimiano que representa todo comportamiento, forma de ver, pensar, actuar y sentir que es exterior a la conciencia. (Durkheim, 1919)
La actualidad de la enseñanza del lenguaje

Tal y como lo apunta el doctor en Filosofía y Letras José Gabriel Marín (2017), el lenguaje se ha enseñado, hasta hace no mucho, a partir de una concepción puramente gramatical, bajo una perspectiva que consistía en

dictar a los estudiantes, mientras estos escribían lo que el docente determinaba, así como trabajos para completar oraciones, colocar acentos, conjugaciones y otras. Al final, la tarea del docente consistía en corregir las faltas de los estudiantes con base en la norma gramatical. (pág. 24)

Esta perspectiva, comenta el autor, deja de lado aspectos fundamentales del aprendizaje del lenguaje, tales como la coherencia, la cohesión, la originalidad de las producciones, tanto verbales como escritas, y el contexto del hablante, aspecto fundamental para tener en cuenta si se desea explorar cómo el educando interactúa con su medio, lo analiza y plantea modificaciones para su estructura.

Este pensamiento es respaldado por Carlos Fernando Vélez (2013), quien hace notar que

el énfasis que los sistemas educativos tradicionales le dan a la memoria y a la repetición está relacionado con la pretensión de construir una 
sociedad estable, estática, basada en dogmas de fe, y no una sociedad cambiante, conflictiva, en tensión permanente, que tiene la posibilidad de transformarse. (pág. 14)

\section{Y añade que}

Hoy asistimos a la extinción del conocimiento localizado, incluso personalizado en la figura del maestro, la institución educativa y sus libros $y$ al surgimiento del conocimiento deslocalizado, disponible de múltiples modos, en todo lugar y en cualquier momento. En esta nueva forma de relacionarnos con el conocimiento, no tiene mucho sentido la memorización mecánica e irreflexiva y cobra importancia estratégica el manejo de la información, para lo cual es indispensable el desarrollo del pensamiento. (pág. 14)

Ante una reflexión similar a esta, a partir de 1990 se suscitó una nueva tendencia en el estudio del lenguaje y su enseñanza, orientada por autores como Gunther Kress (1997) en Estados Unidos, James Gee (1990) en Reino Unido, y Daniel Cassany (1993) en España. Esta tendencia se centró, precisamente, en la contextualización del proceso de formación del estudiante, a partir de una serie de estudios conocidos como New Literacy Studies (NLS, por sus siglas en inglés), los cuales proponían la literacidad como concepto de integración de la enseñanza del lenguaje con su contexto. De acuerdo con Cassany (2006, citado en Gamboa, et. al, 2016)

la literacidad es entendida como la referencia a las prácticas letradas desde la concepción sociocultural, implicando el desarrollo de la competencia de criticidad para el análisis de los discursos, con el propósito de generar impacto tanto en el sujeto como en su contexto. (pág. 58)

En otras palabras, la literacidad es toda aquella práctica de lenguaje que se relacione con el aspecto sociocultural del individuo, y que le permite a este analizar críticamente todo constructo argumental que se le presente, a fin de participar en su desarrollo y afirmación, así como en su refutación y eliminación.

Así pues, el hecho de que exista esta característica en la enseñanza del lenguaje permite brindarle a esta un giro a su intención primordial, para que, en lugar de limitarse a traspasar las normativas de gramática y ortografía para repetir secuencias de construcción de oraciones, le permita al estudiante interpretar, discriminar y comentar críticamente, los elementos discursivos que se mueven a su alrededor, formando, por supuesto, su pensamiento crítico. 
Caracterización del pensamiento crítico

Para entender el pensamiento crítico como concepto, es preciso tener en cuenta que, así como con el lenguaje, este posee un sentido polisémico, que puede ir desde el mero hecho de formularse una opinión sobre cualquier cosa, pasando por el de comentar de forma negativa y premeditada sobre algo, hasta el de generar una opinión exclusivamente personal, basada en un proceso de reflexión previo que se hace sobre aquello que se analiza. (Morales, 2014)

Esta última noción conduce, inevitablemente, a los postulados filosóficos de Immanuel Kant, quien fue, dentro de la filosofía moderna, el gestor del pensamiento crítico, representado de manera práctica en sus tres Críticas, a la razón pura, a la razón práctica, y al juicio. Esto supuso un cambio en el paradigma que impulsaba a los pensadores previos, que se centraba en dar explicación al conocimiento humano, es decir, hallar de manera racional las causas y/o factores que dan lugar al proceso cognitivo del ser desde la noción abstracta de las ideas.

Kant, por su parte, evitó filosofar sobre el conocimiento, a la par que cuestionó la validez de todas las posibles fuentes que pudieran darle origen. Así pues, la revolución que supuso la obra kantiana para el estudio filosófico radica en la propuesta que revela la falibilidad de todo lo que se consigue a través de un proceso $a$ priori, lo cual da las primeras bases de "un conjunto de operaciones cognitivas mediante las cuales buscamos cierta garantía de identidad entre lo que consideramos cierto y la realidad a la que se refiere ese pensamiento que estamos valorando" (Vélez, 2013).

A su vez, el pensamiento crítico se puede precisar a partir de lo que Harry Reeder y Germán Vargas (2009) definen como una "decisión correlativa de formarse como yo verdadero, libre y autónomo en la correlación de la persona individual con la comunidad” (pág. 25).

Este aspecto es particularmente importante si se toma en cuenta el fenómeno que describe Richard Epstein (2006), en el cual existe una cantidad desmedida de información que circula a través de múltiples medios y canales. Ante este escenario, el pensamiento crítico no solo opera como barrera de seguridad contra ideas potencialmente nocivas ${ }^{2}$, sino como fundamento para toda interacción ya que se establece, de manera primordial, el hecho de aplicar crítica a todo elemento

\footnotetext{
2 Al usar el término nocivas, se hace referencia a aquello que perjudique el bienestar del sujeto individual únicamente, ya que la diversidad de los intereses humanos hace complicada la unanimidad conceptual de lo que es beneficioso o nocivo para un grupo social.
} 
ideológico o material que se presente en el ambiente del sujeto.

Habilidades requeridas para el ejercicio del pensamiento crítico

De acuerdo con las propuestas de Jacques Piette (1998), el pensamiento crítico requiere algunas habilidades para ser aplicado en el entorno. El autor propone tres categorías. $\mathrm{La}$ primera refiere a toda actividad capaz de ordenar la información a fin de hacerla transparente, es decir, clara y comprensible.

Dentro de esta categoría, el sujeto debe ser capaz de hacer preguntas respecto del objeto de análisis, de crear sus propias definiciones a partir de su ejercicio racional, de emitir juicios respecto a otras definiciones, y de simplificar a una unidad mínima comprensible todo lo que componga un sistema argumentativo, de un problema, de una situación o de una tarea, a la vez que identifica y aclara los problemas que estos presenten.

La segunda categoría se centra en aquellas habilidades que permiten al individuo construir juicios objetivos acerca de la veracidad misma de la información que ha analizado. Esto es, detalladamente, ser capaz de juzgar la credibilidad de una fuente de información, identificando los presupuestos que esta pueda contener, a la par que juzga la validez lógica de la argumentación que la sustenta.

Este paso da pie a la tercera categoría, la cual se enfoca en las habilidades relacionadas con la capacidad de evaluar los datos, lo cual implica obtener conclusiones apropiadas, a la vez que construye concepciones generales al respector, infiere, formula hipótesis, genera y reformula toda la información de manera personal.

\section{Panorama del pensamiento crítico en la educación}

Considerando todos los elementos definitorios presentados previamente, tanto los referidos a educación, como aquellos concernientes al pensamiento crítico, surge la inquietud de si están estos conceptos relacionados entre sí; es decir, si la educación fomenta el desarrollo del pensamiento crítico en sus programas de formación en todos los niveles.

De acuerdo con Gabriela López (2012) "la enseñanza actual se sigue apoyando en un enfoque pedagógico orientado esencialmente hacia la adquisición de conocimientos, por medio de la enseñanza de asignaturas escolares básicas” (pág. 42). Esto significa que el modelo de enseñanza-aprendizaje más difundido y practicado es aquel en el que prima la transmisión (unilateral por lo general) de temarios en crudo, en forma de 
asignaturas poco especializadas, que ofrecen vistazos generales de multiples ramas del saber humano, sin contexto de aplicación en la realidad, y con énfasis en la memorización y replicación de los contenidos temáticos, dando poco o nulo espacio al debate y discusión de dichos compendios, actividad primordial en el ejercicio del pensamiento crítico. López (2012) continúa afirmando que

se piensa que un buen dominio de la lengua hablada y escrita, el aprendizaje de nociones matemáticas, la adquisición de conocimientos en historia, en geografía, entre otras disciplinas, garantizarían el desarrollo intelectual potencial de los alumnos. Sin embargo (...) aunque el conocimiento es esencial para el desarrollo del pensamiento, esto no garantiza el desarrollo de un pensamiento crítico. (pág. 42)

Prueba de este argumento es el resultado de la investigación de Pineda et.al (2020), la cual indica que, en un espacio académico como lo es una institución universitaria, cerca del 50\% de los docentes y estudiantes ocupan la mayor parte de su tiempo en línea en redes sociales, lo cual permite a los autores afirmar que existe "una primera problemática en la información de los encuestados: a pesar de que usan las redes sociales para comunicarse o divertirse, constituye indirecta- mente su principal mecanismo de información” (pág. 199).

Esto, a su vez, produce, según las conclusiones del estudio, un "nicho favorable para la expansión de fake news (noticias falsas)", dado que cerca del $45 \%$ de los estudiados no se toma el tiempo de leer y verificar la información que recibe a través de los medios electrónicos, conformándose (generalmente) con los titulares, a la par que un porcentaje similar comparte dicho contenido de manera inmediata.

En otras palabras, se confirma que, a pesar de poseer formación académica, un considerable grupo de académicos ${ }^{3}$ carece de formas de pensamiento que los conduzca a la verificación de la información que reciben, así como de interés por la autenticidad de aquello que les presenta, lo cual supone un desafío para los nuevos educadores, si es que estos se proponen el desarrollo del pensamiento crítico en las generaciones venideras.

A fin de llevar a cabo este proceso, Oscar Tamayo, et.al, (2015) indican que existe una intención generalizada de construir una educación mediada por el pensamiento crítico, sin dejar de lado la especialización temática en la cual se desenvuelve

3 El documento hace referencia a académicos cuando hace alusión a docentes y estudiantes por igual, lo cual permite dilucidar la amplitud de la problemática, y su generalización en el espacio de formación. 
el sistema de formación actual. No obstante, advierten que

movilizar el objeto de estudio de la didáctica de las ciencias de la enseñanza -o de la enseñanza-aprendizaje- a la constitución de pensamiento crítico exige nuevas formas de entender las relaciones entre los estudiantes, los profesores y los saberes que circulan en las aulas de clase. (pág. 114)

\section{Pensamiento crítico freiriano}

Así como es inevitable hablar de Kant cuando se evoca el pensamiento crítico, la aplicación de este último en la educación incluye de manera ineludible los aportes del pedagogo brasileño Paulo Freire (1921-1997), quien fue uno de los más notables pensadores latinoamericanos que fomentaron la iniciativa de erigir nuevos modelos educativos, en adición a una revolución de pensamiento cuyo objetivo fuese la emancipación de los patrones antiguos que limitaban el desarrollo de la libertad en los estudiantes, y los condicionaba a obedecer lineamientos de consumo.

En primer lugar, se reconoce la prioridad del diálogo en las relaciones que establecen los miembros del sistema educativo. En palabras del propio Freire, "el diálogo es el encuentro amoroso de los hombres que, mediatizados por el mundo, lo "pronuncian", esto es, lo transforman y, transformándolo, lo humanizan, para la humanización de todos". (Freire, Extensión o comunicacion, 1979).

Este proceso de comunicación y humanización es el mecanismo que sugiere Freire para cumplir con el primer requerimiento del pensamiento crítico, el cual consiste, tal como se mencionó previamente, en formular preguntas acerca del tema o situación, con la adición de considerar las posiciones $y$ sentires de todo ser involucrado en el desarrollo e historia del sujeto de manera cordial, bajo el axioma de que prima el "encuentro amoroso" con la intención de generar nuevos saberes que contribuyan a la liberación.

Respecto al segundo criterio que fundamenta el pensamiento crítico, Freire postula una de las premisas claves de su filosofía, consiste en la aplicación real de los conceptos tocantes a la emancipación de los oprimidos.

La liberación -indica el pensador - no puede darse sin embargo en términos meramente idealistas. Se hace indispensable que los oprimidos, en su lucha por la liberación, no conciban la realidad concreta de la opresión como una especie de "mundo cerrado" (en el cual se genera su miedo a la libertad) del cual no pueden salir, sino como una situación que sólo los limita y 
que ellos pueden transformar. (Freire, La pedagogía del oprimido, 1968, pág. 39)

Esto, puesto en términos de Piette, es la capacidad del sujeto de construir juicios sobre la realidad en la que viven, capacidad que, por demás, se ve coartada en los modos educacionales del último siglo. Al seguir las indicaciones de Freire, los educandos emiten veredictos sobre el estado de sus situaciones vitales, y sobre si estos satisfacen no solo sus necesidades, sino sus aspiraciones como individuo.

Por esta misma razón, el brasileño concluye que "es fundamental entonces que, al reconocer el límite que la realidad opresora les impone, tengan, en este reconocimiento, el motor de su acción liberadora." (Ídem).

Como punto culmen, Freire propone que el currículo sea construido por los propios participantes en el proceso de enseñanza y de aprendizaje, lo cual se corresponde con el propósito del pensamiento crítico, que todos los individuos no solo reconozcan las carencias y potenciales de su entorno, sino que los modifiquen a partir de sus experiencias, y con base en sus ideales, respetando siempre el dialogo amoroso establecido en principio.

Con esta guía filosófica y pedagógica, es posible abordar el pensamiento crítico en la educación, independientemente de su rama de acción, y proponer estrategias concretas que fortalezcan el conocimiento $y$ crecimiento individual y social de las poblaciones que más lo requieran.

\section{El desafío particular en la enseñanza del lenguaje}

Habiendo establecido el reto de encontrar estas nuevas formas de interacción dentro del contexto educativo, cabe preguntarse cuáles serían estas maneras, una vez halladas y aplicadas en el ejercicio específico de la enseñanza de lenguaje.

Tal como se mencionó previamente, las estructuras de aprendizaje se centran en la transmisión de estructuras replicables y de reglas gramaticales y de ortografía que se evalúan de acuerdo con la rigurosidad de uso en ejercicios concretos de habla y escritura, por lo que, si el ejercicio de enseñanza del lenguaje se encaminará hacia la aplicación del pensamiento crítico, deben encontrarse mecanismos para mancomunar estos ejercicios de memorización y mecanización, cuya importancia no es desmeritada en ningún momento ${ }^{4}$, con los postulados

\footnotetext{
${ }^{4} \mathrm{Al}$ realizar esta afirmación no se pretende defender postulados prescriptivistas respecto al uso del lenguaje. Su propósito se limita a evitar que se desechen los conocimientos teóricos que requieren un grado mínimo de memoria para la apropiación y uso de las estructuras lingüísticas.
} 
críticos que exige el nuevo pensamiento.

Por sí sola, esta labor ofrece algunos obstáculos. En primer lugar, está la ya conocida carencia de requisitos mínimos de uso del lenguaje, la cual es explicada por Irma Munguía (2015) al afirmar que

los alumnos no son capaces de elaborar y redactar una tesis de licenciatura que cumpla con las exigencias propias de un escrito de este tipo, como son la claridad, la coherencia, la argumentación adecuada, la fluidez, el manejo apropiado del léxico, entre otras. (pág. 5)

Si bien este argumento hace explicita una carencia del sector universitario, es sencillo extrapolar la afirmación a todos los niveles educativos, dado que los resultados de pruebas estatales (como las ICFES en Colombia) han demostrado un pobre nivel en el uso del lenguaje para la escritura de textos en general, sin importar la tipología o la extensión exigida.

Esto supone un problema por una razón simple. Si no se tiene conocimiento del objeto de estudio, no se puede trabajar críticamente sobre él, pues se carece de la primera habilidad requerida para el proceso de pensamiento, consistente, tal como se mencionó líneas atrás, en realizar preguntas con base en premisas concretas del objeto.
El segundo obstáculo se deriva de la problemática presentada líneas atrás, sobre la considerable cantidad de individuos que reciben y comparten información sin realizar análisis previos respecto a esta. Este hecho genera el fenómeno de la falacia ad populum, que se caracteriza por "la justificación (...) mediante la práctica mayoritaria de una acción determinada, considerándola válida con independencia de su corrección, legalidad o moralidad" (Portillo, 2018, pág. 450), generalmente acompañado de una falacia ad hominem, definida como aquella que da validez a un argumento con única base en su emisor.

La consecuencia de este obstáculo radica en el hecho de que, en contra de los principios del pensamiento crítico, el flujo de la información se incrementa bajo premisas sin fundamento ni cuestionamientos que validen su veracidad, ya que permite la evasión incólume del trabajo implicado en el acto de pensamiento critico. Esto es explícitamente claro en los fenómenos digitales conocidos como cadenas, en las cuales un sujeto replica un contenido (poco veraz por lo general) solo porque los demás a su alrededor lo hacen, y por lo tanto, debe ser correcto (ad populum), y porque lo originó alguien que ostenta una posición socialmente aceptada (ad hominem). 
El tercer obstáculo que presenta la tarea consiste en un fenómeno presentado por el doctor Francisco Villamarín (2018), en el que explica, a partir de la teoría sociología de Durkheim, el por qué las nuevas generaciones tienen poco o nulo acercamiento a la actividad lectora.

A diferencia de las investigaciones tradicionales, que adjudican este hecho negativo a factores como la falta de interés de los jóvenes hacia la lectura, la pereza o el aburrimiento que les produce esta actividad (Carolino, 2009), o a la prioridad que la población joven concede a actividades como escuchar música, chatear por internet y ver televisión, según lo indica la Universidad del Nariño (2011); el autor indica que

esta clase de patologías también se pueden atribuir a un Estado que no piensa, ni reflexiona, ni opera como cerebro social, y que se encuentra alejado y desinteresado de su cuerpo y de su base, que es la sociedad civil y los grupos que la conforman. (Durkheim, 2006, citado en Villamarín, 2018)

El hecho de que sea el Estado un actor responsable del desarrollo de la conducta lectora de los estudiantes, da paso a justificar la idea de desarrollar desde el lenguaje un pensamiento crítico que contrarreste la notoria apatía del cuerpo de gobierno en fomentarlo, ya que la baja actividad lectora contribuye a reforzar los dos obstáculos mencionados previamente, creando un círculo de retroalimentaciones que se expande a medida que los dos elementos que lo conforman (baja lectura y difusión de información falsa) se complementan entre sí.

\section{Posibles Estrategias de Implementación del Pensamiento Crítico por medio de la Enseñanza de Lenguaje $^{5}$}

A partir de los impedimentos especificados, se crea la oportunidad de generar contramedidas pedagógicas que permitan la disminución (o eliminación) de estos factores negativos, a partir de la inclusión de aspectos del pensamiento crítico en la formación del lenguaje, de acuerdo con las diferentes etapas del sujeto. Se advierte, de acuerdo con lo anterior, que un óptimo desarrollo del pensamiento crítico en los educandos se logra con trabajo continuo a lo largo del crecimiento intelectual del individuo.

\section{En la primera infancia}

De acuerdo con los estudios en pedagogía infantil enfocada en el desarrollo del lenguaje, y al

\footnotetext{
${ }^{5}$ Las siguientes apreciaciones se aplican, principalmente, a la enseñanza en países latinoamericanos, ya que estos presentan los inconvenientes descritos, y los modelos educativos de otras regiones, como la europea, poseen otros mecanismos de enseñanza-aprendizaje.
} 
margen de las múltiples posiciones académicas y escuelas pedagógicas que debaten acerca de cómo se produce realmente la concepción del lenguaje en los infantes, es posible definir cinco etapas, que abarcan de los 0 a los 6 años, en los cuales empieza a erigirse un proceso que comienza en el uso de la palabras simples, atendiendo a las necesidades básicas, y que concluye en el desarrollo casi absoluto del lenguaje completo, que se puede ocupar para el análisis del entorno (Peralta, 2000).

Es esta última etapa la que es más recomendable para el fomento del pensamiento crítico, debido a la tendencia del infante a cuestionar su entorno, a preguntar la razón de ser de todo lo que se encuentra a su alrededor, y de indagar por la naturaleza de todo. El análisis empírico suele evidenciar que, durante la crianza, los individuos más grandes, mayores, suelen interrumpir (bruscamente, en muchos casos) esta intención curiosa, debido a que resulta incordiante, o a que las preguntas realizadas amenazan algunos tabúes sociales. Por otro lado, sucede que, en caso de no interrumpir la pregunta, la respuesta a esta deja insatisfecho a quien ha formulado el interrogante, pues suele ser laxa y sin profundidad.

Como alternativa, a fin de interiorizar en el niño o niña que sus preguntas no solo son válidas, sino interesantes y de utilidad para su formación y aprendizaje, se recomienda que, durante la enseñanza del lenguaje (que en esta etapa suele enfocarse en estructuras gramaticales simples, como la oración), se tomen en cuenta todos los cuestionamientos que surjan como interrogantes válidas, dejando de lado los prejuicios que puedan surgir en el docente. Además, las respuestas que se brinden no deben ser inventadas, sino tener sustento argumentativo en el conocimiento académico del maestro o instructor. En caso de no conocerlas, por supuesto, es recomendable hacerle saber al infante que el conocimiento siempre se puede construir en conjunto, por lo que un proceso pequeño de investigación puede solucionar el vacío de conocimiento, sobre todo en la época actual, donde la información se encuentra al alcance de las masas. De esta manera, la práctica de cuestionar aquello que no se entiende se afianza en el sujeto, lo cual crea de manera natural un pensamiento crítico, al eliminar la mala praxis de creer en las falacias, al tiempo que ejercita la primera habilidad que requiere un pensador crítico.

Paréntesis: El Uso de La Transposición Didáctica y el Juego como Elemento de Enseñanza. Es posible que se perciba como una dificultad el hecho de abarcar información de carácter técnico como respuesta a las dudas infantiles que pueden 
originarse durante la enseñanza, pues los niños suelen carecer de estructuras mentales que comprendan ese tipo de complejidades. No obstante, como alternativa, el francés Yves Chevallard (1995) propone una estrategia, la transposición didáctica, que, en síntesis, propone la transformación del contenido curricular, de tal manera que pase de ser un corpus complejo, a un elemento comprensible, sin perder su sentido original. Adicionalmente, se recomiendan los postulados del neerlandés Johan Huizinga (1972), los cuales presentan razones y aplicaciones de las estrategias lúdicas como elementos de construcción social, las cuales facilitan el proceso de transposición.

\section{En la educación básica}

Cuando el estudiante ha alcanzado el nivel de educación básica (que suele rondar los últimos grados de primaria y los primeros de secundaria), su edad se encuentra entre los 8 y los 12 años. En esta etapa, suele ocurrir un fenómeno en educación en el que "se trabaja con muchos supuestos” (Urgilés, 2016, pág. 242). Esto refuerza la idea previamente expuesta de que se pretende que el estudiante adquiera información, y la replique, lo que "genera a veces un activismo desbocado, hacer muchas cosas, planificadas o no, pero sin un norte claro" (Urgilés, 2016, pág. 242). Adicional al activismo mencionado, esta tendencia replica la idea de la falacia ad hominem, siendo el docente esa fuente de conocimiento irrefutable que no se debe verificar. Ante esto, se produce una situación en la que "el alumno utiliza una palabra, pero no sabe lo que ella significa realmente, por ello no puede encontrar ejemplos, porque el ejemplo se vuelve otra abstracción” (Urgilés, 2016, pág. 241).

Por lo tanto, una manera de proponer el pensamiento crítico en esta etapa es modificar la forma en la que se enseñan los contenidos del área -que, por un lado, suelen ser sobre literatura que varía entre la latinoamericana y la española, y por otro, radican en los fenómenos gramaticales como el clásico diptongo, triptongo e hiato-, a fin de convertir los espacios en oportunidades para el diálogo de saberes, el cual se puede definir como "un método cualitativo que busca comprender, sintetizar, teorizar y contextualizar el conocimiento; permite entender los problemas y necesidades que tiene la población, mediante la reflexión y discusión de los actores, basándose en las palabras de la misma población" (Hernandez Rincón et al., 2017, pág. 242). A partir de esta práctica, el estudiante tendrá la capacidad de construir juicios a partir de su conocimiento y sus experiencias, a la par que valida o desmiente los criterios del 
docente y de sus compañeros, lo cual permite cumplir con la habilidad número dos del pensador crítico, así como amenizar y dinamizar los espacios de interacción en el aula.

\section{En la educación media y superior}

Para este punto del desarrollo, se espera que el educando esté en capacidad de abstraer conceptos profundos del entorno que lo rodea, y construir idearios sin referentes materiales. Por lo tanto, el enfoque recomendado para la incorporación del pensamiento crítico es la aplicación del trabajo investigativo de Núñez et.al (2017), el cual sugiere la posibilidad de explorar el conocimiento a partir del Aprendizaje Basado en Problemas, modelo que, según los autores, permite la "construcción de soluciones a problemas basados en la vida real con la finalidad de activar un conocimiento previo $\mathrm{y}$, a su vez, generar un diálogo que permita evaluar críticamente las alternativas” (pág. 88).

Esto entra en contacto con la literacidad, de la que se habló preliminarmente, en el momento en que se pretende usar el contexto real, y actual, del estudiante, para presentarle problemas que serán resueltos a partir de la ocupación retroactiva de sus conocimientos, al mismo tiempo en que explora posibilidades nuevas que amplian su frontera cognoscitiva.
El cómo esta actividad promueve el pensamiento crítico, es explicado por el documento al afirmar que "para generar PC (Pensamiento Crítico) $^{6}$ se requiere un aprendizaje activo. Aprender un concepto para internalizarlo, aplicarlo y observar el valor de lo aprendido para poder evaluar y auto-evaluar como parte integral del conocimiento" (Ídem, p.101), por lo que un trabajo como el que propone este tipo de aprendizaje contribuye significativamente al desarrollo de habilidades no solo de análisis y formulación de juicios, sino de evaluación de criterios, factor que completa la tríada de habilidades que construyen a un pensador crítico, de acuerdo con la teoría antes mencionada.

\section{Conclusiones}

Como se puede notar, el proceso de trabajo con los diferentes niveles de formación presenta inclusiones de los niveles anteriores, ya que la formación es una cadena interminable de procesos, cuyos eslabones dependen en gran medida de los anteriores, especialmente en una forma de enseñanza como lo es el pensamiento crítico.

Como aspecto final, es preciso notar también que el esquema presentado en las páginas anteriores resuelve dos de los tres desafíos planteados, es decir, el relacionado con el poco

\footnotetext{
${ }^{6}$ Paréntesis añadido.
} 
conocimiento del temario, y aquel referido a las falacias que promovían la escasez de procesos críticos. El tercero, alusivo a la falla Estatal en cuanto a la creación y difusión de espacios, herramientas $y$ objetivos que construyan pensamiento crítico en las aulas, no es algo que pueda resolverse con estrategias didácticas.

Queda, por supuesto, en labor de aquellos que componen el sistema aplicar el pensamiento crítico a este aspecto, pues es a través del diálogo crítico y la discusión que se pueden plantear nuevos escenarios en los cuales se transforme la cosmovisión de las nuevas generaciones, aunque, de modo subjetivo, se presente un panorama oscuro en este aspecto, debido a la fuerte existencia e influencia de intereses económicos, políticos y sociales que no pueden explanarse aquí. Así pues, al ser el lenguaje la herramienta más avanzada para la consecución de ideas, y el pensamiento crítico el canal a través del cual se transforma el entorno construido por tal ideario, se ha explicado por qué estas nociones trabajan bien juntas, para brindar al estudiante la oportunidad de ver por sí mismo el mundo, y transformarlo en un lugar mejor para él y para otros.

\section{Referencias}

Carolino, P. (2009). Escribir, leer y aprender en la universidad. Una introducción a la alfabetización académica. Buenos Aires: FCE.

Cassany, D. (1993). La cocina de la escritura. Barcelona: Anagrama.

Chevallard, Y. (1991). La transposición Didáctica. Del saber sabio al saber enseñado. París: Aique.

Durkheim, E. (1919). Las reglas del método sociológico. París: Libraire Félix Algán.

Epstein, R. (2006). Critical Thinking. Belmont: Wadsworth Thomas Learning.

Freire, P. (1968). La pedagogía del oprimido. Nueva York: Siglo XXI.

Freire, P. (1979). Extensión o comunicacion. México.

Gamboa, A., Muñóz, P., y Vargas, L. (2016). Literacidad: Nuevas Posibilidades Socioculturales y Pedagógicas para la Escuela. Revista Latinoamericana de Estudios Educativos 12 (1), 5370.

Gee, J. (1990). Social linguistics and literacies: Ideology in discourses. Reino Unido: Falmer Press.

Hernandez Rincón, H., Lamus Lemus, F., Carratalá, C., y Orozco-Beltrán, D. (2017). Diálogo de saberes: propuesta para identificar, comprender y abordar temas críticos de la 
salud de la población. Salud Uninorte, 33(2), 242-251. https://doi.org/10.14482/sun.3 3.2.10552

Huizinga, J. (1972). Homo Ludens: ensayo sobre la función social del juego. Madrid: Emecé.

Kress, G. (1997). Before writing: Rethinking the paths to literacy. Estados Unidos: Routledge.

López, G. (2012). Pensamiento crítico en el aula. Docencia e Investigación, 41-60.

Marín, J. G. (2017). Enseñanza del lenguaje y nuevos alfabetismos; entre la tradición y la innovación. IE Revista de Investigación Educativa de la Rediech, 21-33. https://doi.org/10.33010/ie_rie _rediech.v7i13.3

Morales, L. C. (2014). El pensamiento crítico en la teoría educativa contemporánea. Actualidades Investigativas en Educación, 1-23.

Munguía Zarataín, I. (2015). El lenguaje y sus problemas de enseñanza. Revista de Ciencias Sociales y Humanidades, 5-9. https://doi.org/10.28928/revist aiztapalapa/792015/ptc/mung uiazataraini

Núñez, S., Ávila, J. E., y Olivares, S. (2017). El desarrollo del pensamiento crítico en estudiantes universitarios por medio del Aprendizaje Basado en Problemas. Revista Iberoamericana de Educación Superior vol. VIII, núm. 23, 84103.

https://doi.org/10.22201/iisue. 20072872e.2017.23.249

Peralta, J. (2000). Adquisición y desarrollo del lenguaje y la comunicación: una visión pragmática constructivista centrada en los contextos. Límite (7), 54-66.

Piette, J. (1998). Una educación para los medios centrada en el desarrollo del pensamiento crítico. En A. Gutiérrez, Formación del profesorado en la sociedad de la información (págs. 63-74). Segovia: Universidad de Valladolid.

Pineda Gómez, H., Jima-González, A., Paradela-López, M., Acevedo Marín, H., Agudelo Velásquez, A., Areiza Villa, S., . . . Tavera Parra, J. (2020). ¿Preparados para las fake news? Un estudio exploratorio de la comunidad universitaria del Tecnológico de Antioquia. En-Contexto 8 (12), 175-204.

Portillo, J. (2018). El uso de falacias en la comunicacion absurda. Logos: Revista de Lingüística, Filosofía y Literatura, 28(2), 443-458. https://doi.org/10.15443/RL28 32

RAE. (20 de Abril de 2021). Diccionario de la Lengua Española. Obtenido de 
Lenguaje:

https://dle.rae.es/lenguaje

Reeder, H., y Vargas, G. (2009). Formación y Pensamiento Crítico. Investigación Educativa y Pedagógica, 23-43. https://doi.org/10.15332/s165 7-107X.2009.0001.02

Searle, J. (1995). The Construction of Social Reality. Londres: Penguin Philosophy.

Tamayo, O., Zona, R., y Loaiza, Y. (2015). El pensamiento crítico en la educación. Algunas categorías en su estudio. . Revista Latinoamericana de Estudios Educativos (11) 2, 111133.

Universidad del Nariño Observatorio Social. (2011). Caracterización social, económica, política, cultural académica de los estudiantes de sociología. Pasto: Universidad de Nariño.

Urgilés, G. (2016). Aula, Lenguaje y Educación. Sophia, colección de Filosofía de la Educación, 20(1), 219-242.

Vélez, C. F. (2013). Una reflexión interdisciplinar sobre el pensamiento crítico. Revista Latinoamericana de Estudios Educativos 9 (2) , 11-39.

Villamarin, F. (2018). Bajos Niveles de Lectura en los Jóvenes Universitarios: Un Problema Estructural. Una Explicación Desde La Teoría de Emile Durkheim. Revista de la Facultad de Ciencias Económicas y Administrativas., 229-241.

https://doi.org/10.22267/rtend. 181902.105 